DOI:

\title{
A shocking enabler of tumour growth
}

The conserved heat-shock response, mediated largely by the transcription factor heat-shock factor 1 (HSF1), allows cells to adapt to stress. Although this response is crucial to protect against some diseases of ageing, including ischaemia and neurodegenerative disorders, Susan Lindquist and colleagues now show that HSF1 has an opposite role in cancer, in which it promotes tumorigenesis.

The authors first showed that $H s f 1^{-/-}$mice were less susceptible to skin tumours induced by the chemical carcinogens dimethylbenzanthracene (DMBA) and 12-O-tetradecanoylphorbol-13-acetate (TPA) than were wild-type mice - tumour latency and survival were increased and tumour incidence and burden were reduced in $H s f 1^{-/-}$mice. The authors also examined the effects of HSF1 on tumours arising in mice heterozygous for the Trp53 mutation $\operatorname{Tr} p 53^{R 172 H}$. As in the skin carcinogenesis model, tumour-free survival was increased in $\mathrm{Hsfl}^{-/-}$mice carrying this allele.

A random sampling of chemically induced skin tumours that developed in $\mathrm{Hsf1}^{-/-}$and wild-type mice showed that all had activating mutations in Hras. To further examine the effects of HSF 1 on transformation by HRAS, the authors expressed an activated form of HRAS, encoded by HRAS V12D,

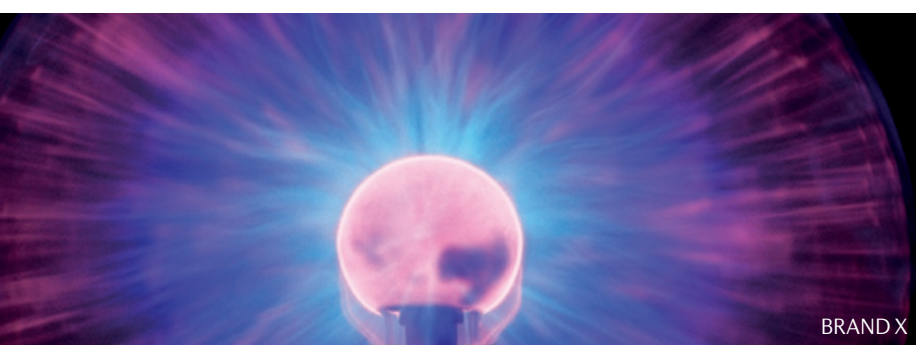

in $\mathrm{Hsf1}^{-/-}$and wild-type mouse embryonic fibroblasts (MEFs) and found that a lack of HSF1 made the MEFs resistant to HRAS ${ }^{\text {V12D }}$-induced focus formation. Similar results were observed using wild-type MEFs expressing short hairpin RNAs (shRNAs) directed against Hsf1.

How does HSF1 promote tumorigenesis? The differential effects of $\mathrm{HRAS}^{\mathrm{V} 12 \mathrm{D}}$ on $\mathrm{Hsf1}^{1^{-/}}$versus wild-type mice were a result of increased proliferation of the wild-type cells, rather than increased death of $\mathrm{Hsfl}^{-/-}$cells. However, expression of MYC or SV40 large T antigen enhanced death in $\mathrm{Hsfl}^{-/-} \mathrm{MEFs}$, so depending on the oncogenic insult HSF1 seems able to promote either proliferation or survival. HSF1 might also affect signalling pathways. The authors found blunted activation of extracellular signal-regulated kinase (ERK) downstream of Ras in the absence of HSF1. Activation of protein kinase A (PKA) downstream of G-protein-coupled receptor activation was also reduced, suggesting that HSF1 can modulate at least two oncogenic signalling pathways.

Yet another effect of HSF1 on tumorigenesis might result from its role in translation. Ribosomal biogenesis normally depends on growth factors, but cancer cells can circumvent this requirement. The expression of ribosomal subunits was reduced in serum-starved $\mathrm{Hsf1}^{-/}$or $\mathrm{Hsf1}$ shRNAexpressing MEFs compared with wild-type cells, indicating that HSF1 deficiency promotes the dependency of the translational machinery on growth factors. HSF1 might also affect translation through its effects on the mammalian target of rapamycin (mTOR) pathway. Inhibition of mTOR or loss of its downstream effector
p70S6K (ribosomal protein S6 kinase, $70 \mathrm{kDa}$ ) impairs protein translation and reduces cell size, and MEFs from $H s f 1^{-/-}$mice had reduced p70S6K phosphorylation and a smaller size.

Finally, HSF1 can promote glycolysis, which occurs in most cancer cells and is beneficial for tumour growth and survival. Glucose uptake was decreased in $\mathrm{Hsf1}^{-/-}$compared with wild-type MEFs, and $H s \mathrm{fl}^{-/-}$cells were less dependent on glucose for survival.

Is HSF1 relevant in human cancers? The authors examined human cell lines of varying malignant potential that were either transformed in the laboratory or derived from human tumours with diverse histological origins and various genetic abnormalities. In all cases, they found that the growth of tumorigenic cells was decreased by shRNA knockdown of HSF1.

HSF1 seems to act not as a classic oncogene, but as a modulator of tumorigenesis, affecting multiple pathways that allow cells with oncogenic defects to adapt and survive. Activation of HSF1 is being explored for treating ischaemic injury and some neurodegenerative diseases, and it needs to be determined whether such therapies might enhance tumorigenesis if used in the long term. Furthermore, any potential cancer therapeutics that inhibit HSF1 could possibly affect neurodegeneration and ageing. Hopefully a greater understanding of HSF1 will lead to the development of more effective therapeutics for several diseases.

Sarah Seton-Rogers

ORIGINAL RESEARCH PAPER Dai, C

Whitesell, L., Rogers, A. B. \& Lindquist, S. Heat shock factor 1 is a powerful multifaceted modifier of carcinogenesis. Cell 130, 1005-1018 (2007) 